\title{
Promoting urban projects through social networks using analysis of users influence in social graph*
}

\author{
Dmitry Donchenko, Natalia Sadovnikova, Danila Parygin, Olga Shabalina \\ Department of CAD \\ Volgograd State Technical University \\ Volgograd, Russia \\ dmitrydonchenko92@gmail.com,npsn1@ya.ru,dparygin@gmail.com,oashabalina@gmail.com
}

\begin{abstract}
In this paper we consider the problem of designing the instruments for promotion of urban projects in social networks and studying approaches to organization of "distributed control" of city development. We suggest a method for organizing synergy of urban communities based on the analysis of influence of subjects in social graph.
\end{abstract}

Keywords—social graph; social networks; breadth-first search; data analysis; development management; decision support; urban projects

\section{INTRODUCTION}

Analysis of social needs and their consideration in strategic plans of city development, as well as involving intellectual potential of population are important conditions for achievement of planned results in the process of implementation of strategic city development [1]. For creating self-organizing mechanisms of the urban system it is necessary to involve citizens in the resolution of issues related to the operation and transformation of the urban environment. The regulation of public relations, conducting competent information policy, creating conditions that motivate people to participate in the reformation, allows not only to expand the circle of participants in the decision-making process, but also to raise the level of trust in the government [2].

In the matter of the establishment of mechanisms for the promotion of socially oriented projects it is becoming important to use the transformative power of social media. In practice, this means finding effective ways to connect people to information, the organization of cooperation, the process of two-way communication and innovation [3].

In recent years popular social networks such as Facebook, Twitter, VK et al., have managed to become an important part of our society and have become a powerful political, economic and social tool. Social networks have been playing a significant role in shaping the outlook of people and spreading new trends and opinions. Popular social networks can be used for creating the mechanisms of organization of urban communities, providing the basis for motivated activity by the reformation of the city.

Creation of sense of belonging of the citizens to the results

The study was financially supported by RFBR, research projects No. 1607-00353_a, No. 16-07-00388_a. of the reforms should be the first step. On the other hand, it is possible to monitor the reaction of urban communities to certain changes, by taking into account the emotional reactions in the network. A decision support model that can be used to assess the various city services and keep track of the dynamics of development of the city can be created based on analysis of this network [4].

Thus, the use of social media to promote socially important projects can be considered as necessary condition for improvement of effectiveness of management decisions [5]. A key component of social media is the community of common social network users who receive and transform the information and shape communication streams. Activity of such users (so called actors) and their involvement in the communication define their "weight", which is the actual measure of their influence on other actors. This type of social leaders will be called further "changing agent" [6].

Changing agents, joint by discussed problem, and actors related with them form social graph.

Social graph is usually used as a social network model. The term "social graph" means a graph where vertices are social objects, which can be user profiles with various attributes (such as name, city of residence, date of birth, etc.), user communities, media content (pictures, videos, text, etc. posted by users of social networks), and the edges are relations (social connections) between the users.

In this paper, we consider a social graph as a model of social network. Vertices of this graph are the users of this social network, and edges appear to be social relations between the users, which are expressed in a variety of ways of interaction between the users (it depends on exact social network. For example, such interactions kind of relations for social network Twitter are represented by reposts of messages, which are placed by other users). The edges of social graph are oriented. Weight of the edges characterizes the intensity of social connections between users.

Social graph may form a variety of information streams spread with different sources. Therefore, with proper information management process, it is possible to influence the choice of the activities of people and provide support for positive initiatives. To do this, it is necessary to identify ways 
to influence and develop mechanisms to promote the right ideas.

\section{TECHNOLOGY OF PROMOTION URBAN PROJECTS IN SOCIAL NETWORKS}

To identify the major problems of the city and to promote ideas to solve them it is especially important to ensure close interaction between decision makers and the population as the target group for their decisions. Social networks can significantly facilitate such cooperation, to involve in discussions a number of interested parties and arrange direct discussion of urban problems. Moreover, the social networks can also be used to determine the most pressing problems in the city. Problems which heavily affect the life of citizens can be identified by analyzing trends which are popular among people living in the area.

Thus, social networks can serve as a tool to identify the main problems of the city and promote ideas to address them. We propose the technology that includes the following steps:

1) Identification of urban problem and discovering possible growth areas;

2) Identification of changing agents;

3) Network configuration:
a) Creation of new actors;
b) Creation of new connections;
c) Creation of changing agents;

4) Management of dynamic goal setting with the situation of changeable actors' objectives:

a) Creation of a situation of objectives refocusing;

5) Generation of events motivating population to improve the urban environment;

6) Adaption of technologies for obtaining resources for new projects;

7) Analysis of degree of objectives achievement.

\section{IDENTIFICATION OF URBAN DEVELOPMENT PROBLEMS}

In this paper, we propose an approach for dynamic elicitation of the most urgent urban problems through the analysis of social networks. The algorithm of its realization can be represented by the following sequence of steps:

1) Gaining (with the use of social networking tools) information about the most important trends among residents of the city, belonging to the category "city".

2) Building a social graph of users of social networks, participating in the discussion of a specific topic.

At this stage, social graph is created, whose vertices are the users of social networks, and edges are the relationships between the users, which are related to a particular topic (examples of such relationships - "likes", "reposts", etc.). The edges of the graph are oriented from the user, whose post on the subject has received a response, to the users, who react to this post.

3) Analysis of relationships between users in a social graph.
At this stage, we define how important and relevant analyzed topic is. We propose to use the density of the graph as a measure; it is given by the following formula:

$$
D=(2 *|E|) /(|V| *(|V|-1))
$$

$$
\begin{aligned}
& D-\text { the density of the graph; } \\
& E \text { - the number of edges; } \\
& V \text { - the number of vertices. }
\end{aligned}
$$

Thus the higher the number of connections between users is, the higher the density of the graph is. Sparse graph means that the subject is not widely discussed among the subjects of created social graph, and at this stage it should be assigned a lower priority compared to other subjects.

4) Analysis of the social graph subjects and search of changing agents.

After determining the most relevant topics the problem of determining the subjects, influenced most strongly on the promotion of these topics, appears. This task is not always obvious, because hidden changing agents can be often those users who do not have great popularity in social networks, but who bring their ideas right in time to the obvious agents of influence. Obvious changing agents, in turn, have a large number of subscribers, and, as a result, opportunities to promote their ideas.

\section{DEFINITION OF “INFLUENCE” IN SOCIAL NETWORK}

To answer the question of how to identify the most influential users of social networks, it is first needed to define the term influence. The more the user influences opinions of other users the more influential he considers. In addition, the user's influence to a great extend determines his possibility to disseminate specific information among other users and extend the audience, which he is able to reach. Social influence appears when a person adapts his behavior, attitude and opinion in certain things to the behavior and attitudes of other people in a social system [7]. Influence of people on each other in the society was the topic of many marketing research since the 1960 's. Influence does not necessarily imply direct interaction between two people, it is mostly based on information about other people [8]. In online communities, information is transferred between the community users in the form of digital content. Thus, we can make conclusion about the type of social influence, typical of social networks: a measure of the social network user's influence is the number of users who change their behavior in a social network in response to changes in the behavior of this user, and also the frequency with which they do it.

\section{ALGORITHM OF MEASURING USER'S INFLUENCE}

As already mentioned, the influence does not often require direct interaction between the subjects of the social graph. Thus, when creating an algorithm to determine the influence of the subject of the social graph is important to consider not only its direct, but also indirect links with other subjects. 
Another thing to take into account while determining the subjects of the social graph influence is to consider the relevance of accomplished social interactions. Currently, most of research examines the impact on the basis of static attributes, such as number of friends and followers in the social network, the number of tweets and retweets, mention and answers in the activities column. All these studies make implicit assumptions about the underlying dynamic process in which the social network users can influence only their friends and followers, with an equal degree of influence and the same speed of propagation; in other studies the impact is considered to be a "random walk" on the corresponding static network [7]. According to the theory of diffusion, information cascades come from community leaders to their followers. In the majority of the diffusion models distributors have a certain probabilities to influence their followers, who also have a certain threshold at which they are sensitive to influence. The main goal of most of the diffusion models is finding the community leaders, who have the greatest influence on their followers. These methods are the basis for the so-called viral marketing, the purpose of which is to activate the effect of a chain reaction and, thus, the greatest coverage of the audience at the lowest price [9].

Summarizing these criteria, we propose to divide solution to the problem of calculation the influence of subjects of social graph into two steps:

The first step is to determine the weights of the edges of social graphs. For this purpose it is necessary not only to calculate the intensity of social connections between the joined vertices, but also to take into account time relevance and consistency of these interactions, and calculate the probability of occurrence of such interactions in the future. Thus, after the first step we obtain a social graph whose vertices are connected by directed edges that have a weight which determines the degree of influence of one vertex to another.

The second step is calculating vertices' weights, which reflects influences of these vertices within the whole system. Thus, the weight of each vertex must be calculated based not only on the weights of outgoing edges, but also taking into account the weights of the edges outgoing from the vertices associated with the followers of the initial vertex. In this case, the sum of weights of outgoing edges of vertices associated with "followers" of initial vertex has less affect on the influence exerted by the initial vertex throughout the system. Thus, the proposed algorithm must also take into account the "damping" factor that will determine the coefficient for calculating the sum of the weights of edges of vertices which are not in direct connection with the initial one.

These requirements allow the algorithm to be compared to the known Page-rank algorithm. Page-rank algorithm also takes into account the damping factor. Page-rank implies the existence of "random surfer", who visits a particular web page, and randomly keeps clicking on the links located on this page. Damping factor is the probability that a random visitor becomes bored at some point and requests another web page. In various studies different values of damping factors have been tested, but in most practical cases the value of this factor is adjusted to about 0.85 [10].

\section{APPLICATION OF THE ALGORITHM}

In this research, we presume that the damping effect is defined as a measure of reducing the influence of the social graph's subject in relation to the subjects, who are not in the direct connections with it, but have mutual friends, with which they actively interact. Assume the existence of the random user of social network Twitter, who appeared in a profile of another user of social network randomly selects a user profile page, reposts which produced the current user accounts. Thus, the damping effect is the probability that a random user requests another randomly selected profile. Like in Page Rank algorithm, we take this factor equal to 0.85. Algorithm for calculating weights of vertices social graph, using these criteria is shown below:

$$
W n=d^{1} \sum_{i=1}^{i=N_{1}} W e_{i}+d^{2} \sum_{i=1}^{i=N_{2}} W e_{i}+\ldots+d^{C \max } \sum_{i=1}^{i=N_{C \max }} W e_{i}
$$

$C$ - the number of edges between vertices (vertex removal rate);

Cmax - maximum degree of removal of the vertex;

$N c$ - number of edges outgoing from the vertex with degree $C$;

$W n$ - vertex weight;

$W e_{k}-$ the weight of the edge;

$d$-damping factor, value 0.85 ;

$k$ - serial number of the edge (from a total number $N c$ ).

For graph traversal the breadth-first search algorithm is used. List of adjacent vertices was selected as the data structure for storing the graph, because this structure compared to the adjacency matrix greatly reduces memory costs for the implementation of the algorithm. So the complexity of the algorithm for each vertex in the graph is $\mathrm{O}(N+M)$, where $N$ is amount of vertices, $M$ is amount of edges.

Thus, it is possible to evaluate the complexity of the algorithm for all vertices of the graph like $O(N *(N+M))$.

Consequently with large amount of vertices the computational complexity of the algorithm is quite high. In this case since the breadth-first search is fast enough (running in linear time) algorithm, if the graph is stored as a list of adjacent vertices, the best solution to reduce the computation time of the algorithm is its parallelization. 


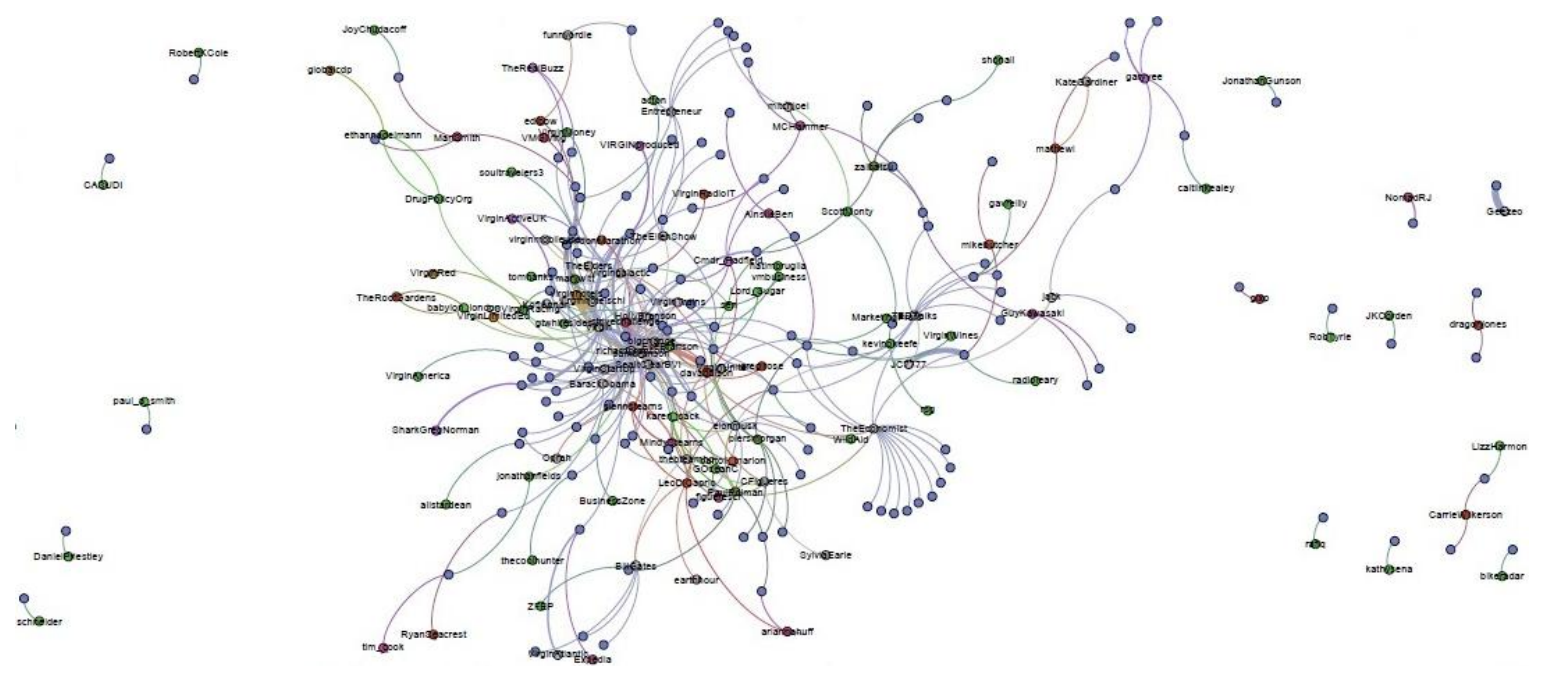

Fig. 1. Visualization of social graph using Gephi software

As a result the algorithm calculates the weight of each vertex, which is a measure of the influence of the user, who is represented by this vertex. In this case the algorithm takes into account not only the user's influence on the users, who are his friends, but also, the impact of these users to their friends, and so on.

As an example, Twitter social network was used to test this approach. The number of reposts made by users of this social network was selected as a metric to calculate the weights of the edges. API of social network Twitter was used to build a social graph Fig. 1. Result of calculation of subjects influence in social graph is shown in Fig. 2.

\section{CONCLUSION}

In this article we suggested an approach of creation of mechanisms to promote socially significant urban projects based on social network.

The basic stages of solving this problem are presented. The method for the detection of urban development problems on the basis of calculation of the density graph, which allows users to take into account the activity, is described. The algorithm for determination of hidden changing agents of subjects in social graph is developed. An example of weight calculation is shown.

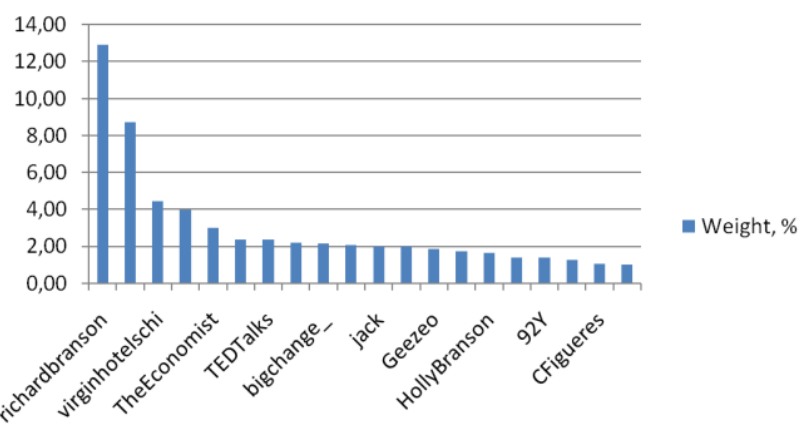

Fig. 2. Calculation of subjects influence in graph with Richard Branson
Nevertheless, these ideas need further investigation. In particular it is necessary to develop a method for configuring a network that will allow solving the problems associated with goal-setting and generation of pulses to promote new urban development ideas.

\section{REFERENCES}

[1] N.P. Sadovnikova, D.S. Parygin, E.P. Gnedkova, B.Kh. Sanzhapov, and N.P. Gidkova, "Evaluating the sustainability of Volgograd," The Sustainable City VIII, Proc. of the 8ht Int. Conf. on Urban Regen. and Sustain., Sec. 3, Putrajaya, Malaysia. WIT Press, 2013, pp. 279-290.

[2] N.P. Sadovnikova, B.Kh. Sanzhapov, D.S. Parygin, N.P. Gidkova, and E.P. Gnedkova, "Participative management of city development. Implementation mechanisms on the basis of information technologies," Biospheric compatibility: human, region, technologies, No. 3, 2013, pp. 80-85.

[3] "Social media and the city," 2012, https://www.ibm.com/smarterplanet/global/files/uk_uk_en_none_file 1_488kb.pdf

[4] Y. Zheng, L. Capra, O. Wolfson, and H. Yang, "Urban computing: Concepts, methodologies, and applications," ACM Trans. Intell. Syst. Technol, vol. 5, No. 3, 2014.

[5] "The use of social media to involve citizens in urban mobility projects and city planning," Policy note: CIVITAS Cleaner and better transport in cities, 2015, http://eltis.org/sites/eltis/files/trainingmaterials/civ_polan3_m_web.pdf

[6] X.S. Briggs, "Social capital and the cities: advice to change agents," 1997, http://www.hks.harvard.edu/saguaro/pdfs/SKandCities.pdf

[7] Leenders, and Th. A. J. Roger, "Modeling social influence through network autocorrelation: constructing the weight matrix," Social Networks, vol. 24(1), 2002, pp. 21-48.

[8] G. Robins, P. Pattison, and P. Elliot, "Models for social influence processes," Psychometrika, vol. 66(2), 2001, pp.161-190.

[9] L. Jingxuan, P. Wei, L. Tao, and S. Tong, "Social network user influence dynamics prediction," APWeb, LNCS 7808. Berlin Heidelberg: Springer-Verlag, 2013, pp. 310-322.

[10] B. Sergey, and L. Page, "The anatomy of a large-scale hypertextual web search engine," Seventh International World-Wide Web Conference (WWW 1998), Brisbane, Australia, April 1998. 